\title{
Short-Latency Influence of Medial Frontal Cortex on Primary Motor Cortex during Action Selection under Conflict
}

\author{
Rogier B. Mars, ${ }^{1,2}$ Miriam C. Klein, ${ }^{1}$ Franz-Xaver Neubert, ${ }^{1}$ Etienne Olivier, ${ }^{3}$ Ethan R. Buch, ${ }^{1,4}$ Erie D. Boorman, ${ }^{1,2}$ and \\ Matthew F. S. Rushworth ${ }^{1,2}$ \\ ${ }^{1}$ Department of Experimental Psychology, University of Oxford, Oxford OX1 3UD, United Kingdom, ${ }^{2}$ Centre for Functional Magnetic Resonance Imaging of \\ the Brain, University of Oxford, John Radcliffe Hospital, Oxford OX3 9DU, United Kingdom, ${ }^{3}$ Institute of Neuroscience, Université catholique de Louvain, \\ B-1200 Brussels, Belgium, and ${ }^{4}$ Human Cortical Physiology Section, National Institute of Neurological Disorders and Stroke, National Institutes of Health, \\ Bethesda, Maryland 20892-1430
}

Medial frontal cortex (MFC) is crucial when actions have to be inhibited, reprogrammed, or selected under conflict, but the precise mechanism by which it operates is unclear. Importantly, how and when the MFC influences the primary motor cortex (M1) during action selection is unknown. Using paired-pulse transcranial magnetic stimulation, we investigated functional connectivity between the presupplementary motor area (pre-SMA) part of MFC and M1. We found that functional connectivity increased in a manner dependent on cognitive context: pre-SMA facilitated the motor evoked-potential elicited by M1 stimulation only during action reprogramming, but not when otherwise identical actions were made in the absence of conflict. The effect was anatomically specific to pre-SMA; it was not seen when adjacent brain regions were stimulated. We discuss implications for the anatomical pathways mediating the observed effects.

\section{Introduction}

Medial frontal cortex, more specifically the presupplementary motor area (pre-SMA), is important in situations involving the direct competition (Ullsperger and Von Cramon, 2001), inhibition (Nachev et al., 2007), updating (Shima et al., 1996), or reprogramming (Isoda and Hikosaka, 2007) of actions. Previous work has shown that repetitive transcranial magnetic stimulation (TMS) over pre-SMA during response conflict results in a greater activation in the motor cortex controlling the competing response, as indexed by the lateralized readiness potential (LRP) (Taylor et al., 2007), indicating that the pre-SMA is in a position to influence the motor cortex. However, the questions of how and when this influence is exerted remain. Importantly, previous studies could not investigate whether the effect of pre-SMA was predominantly to inhibit the incorrect motor response or to facilitate the correct motor response. Moreover, the limited temporal resolution of repetitive TMS means that the timing of this influence could not be determined. Finally, there has been speculation about the anatomical route by which medial frontal cortex (MFC) influences action selection (Kerns et al., 2004; Isoda and Hikosaka, 2008) and a better understanding of the timing of the influence of pre-SMA might clarify this issue. In the present study, we address these issues by looking at the functional con-

Received March 24, 2009; accepted April 20, 2009.

This study was supported by the Medical Research Council. R.B.M. was supported by a Marie Curie Intra-European Fellowship within the 6th European Community Framework Programme. E.0. was supported by a Royal Society International Incoming Short Visits grant awarded to M.F.R.S. and E.0.

Correspondence should be addressed to Rogier B. Mars, Department of Experimental Psychology, University of 0xford, Tinbergen Building, South Parks Road, 0xford OX1 3UD, UK. E-mail: rogier.mars@psy.ox.ac.uk.

D0I:10.1523/JNEUROSCI.1396-09.2009

Copyright $\odot 2009$ Society for Neuroscience $\quad$ 0270-6474/09/296926-06\$15.00/0 nectivity of pre-SMA with primary motor cortex (M1) using the paired-pulse TMS technique.

We asked healthy human participants to perform a task modeled on the paradigm developed by Isoda and Hikosaka (2007). The task (see Fig. 1a) required participants to either execute a prepared response or switch to another response. A test TMS pulse was delivered over left M1. On some trials it was preceded by a conditioning pulse over pre-SMA. The conditioning pulse can modulate the amplitude of the motor-evoked potential (MEP) elicited in the hand muscle by the M1 test pulse (see Fig. $2 b$ ), providing a quantification of the influence of pre-SMA on corticospinal excitability. We then probed the differential influence of pre-SMA on M1 depending on the cognitive state of the participants, i.e., whether participants executed the prepared response or switched to another response. Previous studies of functional connectivity between dorsal premotor cortex (PMd) and M1 have reported changes time-locked to task events (Koch et al., 2006; O'Shea et al., 2007), suggesting the technique may be suitable for probing the influence of pre-SMA on M1 during cognitive control of action selection.

\section{Materials and Methods}

Participants and experimental setup. Forty healthy volunteers (age 19-40 years) with no personal or familial history of neurological or psychiatric disease participated in one or more of the experiments (approved by the Oxfordshire Research Ethics Committee and conducted in accordance with the declaration of Helsinki): 11 participants ( 7 females) in the switch experiment, 10 (6) in the stay experiment, 6 (3) in the M1-control experiment, 7 (5) in the PMd-control experiment, and 6 (3) in the interpulse interval (IPI) experiment. All participants were right-handed and gave written informed consent. Participants wore tight-fitting bathing caps, 


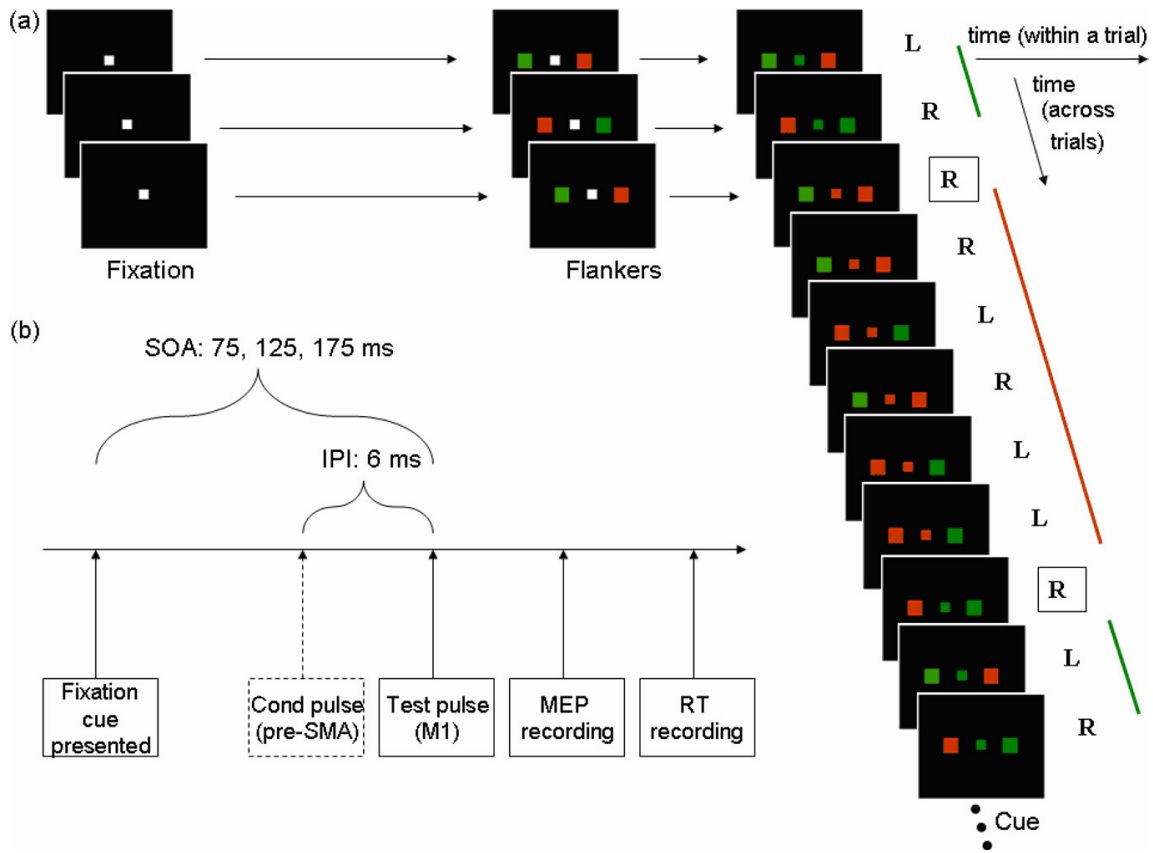

Figure 1. $\quad \boldsymbol{a}$, On each trial of the action reprogramming task participants were presented with a centrally displayed white square. Subsequently, two colored flankers (red and green, sides random) appeared on either side of fixation. Four hundred and fifty to six hundred milliseconds after flanker onset, a central colored cue appeared, to which participants responded with the index finger of the hand on the side with the congruent color. Trials were blocked into groups with the same cue color, so that as soon as flankers were presented, participants could anticipate and thus prepare an action based on the cue color presented in the previous trial. The prepared response would, however, be incorrect when the central cue color changed from one trial to the next (switch trials, boxed letters). Correct actions are indicated by " $\mathrm{R}$ " (right) and " $\mathrm{L}$ " (left). $\boldsymbol{b}$, The M1 test pulse was applied 75, 125, or $175 \mathrm{~ms}$ after the central color cue onset. A pre-SMA conditioning pulse preceded the M1 test pulse by $6 \mathrm{~ms}$ on half of the TMS trials.

on which TMS sites were marked, and earplugs to protect against TMS noise. A chin rest was used to minimize head movements.

Behavioral task. The task (Fig. 1a) required participants to respond with the left or right index finger in response to visual stimuli presented on a screen $\sim 85 \mathrm{~cm}$ in front of them. Each trial began with the presentation of a central white square $\left(4.7^{\circ}\right.$ width) followed by the presentation of flanker stimuli on each side of the central square. The flankers were always one square (width $6^{\circ}$ ) on each side, one of them red, one of them green, with trial-wise random assignment of color to side of display. After a variable delay (450-600 ms, uniform distribution), the central square became either green or red, instructing the participant to respond with the index finger on the side corresponding to the flanker of the same color.

The critical manipulation imbedded in the task was that the central cue took the same color for trains of 3-7 consecutive trials (uniform distribution). This afforded participants the opportunity to prepare, in the period between the onset of the flankers and the onset of the central color cue, the response that was most likely to be required. The manipulation meant that there were two types of trials: stay trials, on which the fixation color was identical to that of the previous trial, thus allowing participants to execute the prepared response, and switch trials, on which the fixation color was different from the previous trial, thus requiring participants to inhibit the prepared response and reprogram a response with the other hand.

Stimuli were pseudo-randomly generated and a different stimulus order was used for each block and for each participant. Custom software written in Turbo Pascal controlled the experiment. Before the actual experimental session, participants were familiarized with the task for 30 trials. The main experiment consisted of 7 (switch and stay experiments) or 5 (PMd and M1-control experiments) blocks of 180 trials each. Each block contained 30 switch and 150 stay trials. The IPI experiment consisted of 5 blocks of 220 trials, each containing 36 switch and 184 stay trials.

Switch and stay experiments. During the switch and stay experiments, TMS was delivered through two figure-of-eight shaped coils, connected to monophasic Magstim 200 stimulators (Magstim Company). Test coil intensity was such that an MEP of 1-1.5 $\mathrm{mV}$ was elicited in the contralateral first dorsal interosseous (FDI) muscle. Conditioning coil intensity was set at $120 \%$ of the resting motor threshold (RMT), which in turn was defined as the minimum intensity, when the coil was over the M1 hotspot, needed to elicit an MEP of $>50 \mu \mathrm{V}$ in the relaxed FDI muscle on 5/10 trials. The IPI between conditioning and test pulses was $6 \mathrm{~ms}$, which has been shown to be effective in a previous study of medial premotor areas (Civardi et al., 2001). The relative frequency of switch and stay trials remained the same in both experiments and, in both cases, TMS was delivered either 75,125 , or $175 \mathrm{~ms}$ after the onset of the central color cue [stimulus-onset asynchrony (SOA)] (Fig. 1b). These times were chosen to cover the period during which pre-SMA neuronal activity changes occurred in the experiment performed by Isoda and Hikosaka (2007) and the times when other premotor areas have been shown to exert an influence over M1 (O'Shea et al., 2007).

The test coil was placed over the position which allowed elicitation of the largest MEP for a given intensity in the FDI muscle of the righthand, with the coil held tangentially to the skull with the handle oriented posteriorly at $\sim 45^{\circ}$ from the mid-sagittal axis. The conditioning coil was placed with the handle pointing in the anterior direction, as close as possible to a position $4 \mathrm{~cm}$ anterior to electrode position $\mathrm{Cz}$, previously shown to be an appropriate location for stimulation of pre-SMA (Rushworth et al., 2002) (Fig. 2a). Coil positions were assessed in nine participants using Brainsight frameless stereotaxy (Rogue Research) (Fig. 2c). Average Montreal Neurological Institute coordinates for the conditioning coil were [ $\left.\begin{array}{lll}4 & 18 & 65\end{array}\right]$ and therefore clearly within pre-SMA (Picard and Strick, 1996). Average coordinates for the test coil were $[-40$ $-1060]$, just anterior to the central sulcus, consistent with previous reports of the hand area of M1. In addition, diffusion-weighted imaging (DWI) was used to further assess the anatomical pathways mediating the observed effects (supplemental Material II, available at www.jneurosci. org as supplemental material).

We first collected pre-SMA/M1 interaction data in two separate experiments involving different participants. During these switch and stay experiments, pulses were delivered almost exclusively on switch and stay trials, respectively. For these two experiments, a total of 14 pulse trials per hand, SOA, and pulse type (single or dual pulses) were delivered and used for the analyses on switch and stay trials, respectively. The presence or absence of TMS could not serve as precue indicating trial identity, because the pulses were only applied after the switch or stay cue had already occurred. However, six instances of pulse application on the opposite trial prevented participants from detecting, as assessed by subsequent report, any relationship between trial type and TMS delivery. For the same reason, each trial type was also presented in the absence of TMS on at least $20 \%$ of instances of that trial type in each block. TMS trials were presented at least 7 (mean 10.5) seconds apart, to ensure that pulses on adjacent trials did not influence each other. In each block, TMS trials were distributed evenly over response hand, SOA, and single- or dualpulse TMS.

For the analysis of the effect of pre-SMA on M1, we thus concentrated on a between-session design: we analyzed TMS data from switch trials in one session (referred to as the switch experiment) and stay trials in a separate session (the stay experiment). This was necessary because probing both an adequate number of switch and stay trials at three different SOAs with both single and paired pulses would have resulted (1) in the 
participants receiving a very large number of TMS pulses and (2) in an exceedingly long experiment.

$M 1$ and PMd-control experiments. We tested the anatomical specificity of pre-SMA/M1 interactions in two control experiments. In the M1-control experiment, we applied TMS on both switch and stay trials at an SOA of $125 \mathrm{~ms}$ through a single figure-or-eight coil, connected to the stimulators via a BiStim module, placed over the hand area of M1. This is a critical control because it tests whether any observed changes in MEPs are caused by the mediating influence of pre-SMA or merely the result of processes internal to M1 (O'Shea et al., 2007).

A second control experiment tested whether pre-SMA TMS effects were caused by spreading of activation from the pre-SMA coil into the adjacent PMd. During this PMd-control experiment, the conditioning coil was placed over the right hemisphere, because the average pre-SMA location was also just within the right hemisphere, at a location $2 \mathrm{~cm}$ anterior and $1 \mathrm{~cm}$ medial to the location in right hemisphere which resulted in the largest MEP in the contralateral FDI for a given TMS intensity ("hotspot"), which has previously been shown to be a reliable landmark for PMd and dorsal precentral sulcus (O'Shea et al., 2007). TMS intensities were the same as in the switch and stay experi-

ments. In both of these control experiments the IPI was $6 \mathrm{~ms}$, as in the switch and stay experiments. These control experiments are thus not comparable with previous paired-pulse TMS experiments probing $\mathrm{PMd} / \mathrm{M} 1$ functional connectivity, which used an IPI of $8 \mathrm{~ms}$ (Koch et al., 2006; O’Shea et al., 2007).

IPI experiment. In a final control experiment, we tested for effects of different IPIs between conditioning pulses over pre-SMA and test pulses over M1. Single pulses and dual pulses at IPIs of 3, 6, 9, 12, and $18 \mathrm{~ms}$ were delivered on switch trials at an SOA of $125 \mathrm{~ms}$. A total of 15 trials were presented for each pulse and hand combination, distributed equally across five experimental blocks. Stimulation parameters were the same as in the other experiments.

Electrophysiological recording and data analysis. MEPs were recorded from the right-hand FDI muscle using $\mathrm{Ag}-\mathrm{AgCl}$ electrodes in a tendonbelly montage. EMG responses were bandpass filtered between 10 and $1000 \mathrm{~Hz}$, with an additional $50 \mathrm{~Hz}$ notch filter, sampled at $5000 \mathrm{~Hz}$, and recorded using a CED 1902 amplifier, a CED micro 1401 Mk.II A/D converter, and a PC running Spike2 (Cambridge Electronic Design).

Analysis of electrophysiological data concentrated on peak-to-peak amplitudes of the MEPs measured on TMS trials. Trials with incorrect or premature [reaction time $(\mathrm{RT})<150 \mathrm{~ms}$ ] responses, those in which the test pulse failed to elicit a reliable MEP (amplitude $<0.2 \mathrm{mV}$ ), and those in which participants precontracted the FDI muscle before application of the conditioning pulse (EMG amplitude $>0.1 \mathrm{mV}$ in the $80 \mathrm{~ms}$ before the pulse) were discarded from the analysis. Grubb's test was used to detect outliers in the obtained values of one block and these were excluded from the analysis. After this preprocessing, on average $11.45(\mathrm{SEM} \pm 0.62)$ and $11.65( \pm 0.67)$ trials were included per condition in the switch and stay experiments, respectively.

To account for differences in coil placement between blocks, MEP sizes were normalized within each block. Analyses of MEPs were performed on the median of the normalized MEP amplitudes in each condition. Analyses of both behavioral and electrophysiological data were conducted using ANOVA tests, using repeated measures where possible. Significant effects were identified based on Huynh-Feldt corrected ANOVA values, using SPSS 15.0. Post hoc paired-samples two-sided $\mathrm{t}$ tests were used to further investigate significant effects in the ANOVAs. Post hoc tests on electrophysiological data were performed on the dual- pulse MEP amplitudes expressed as percentage of the respective singletrial MEP amplitude ("MEP changes").

\section{Results}

\section{Behavioral data}

ANOVAs of RTs on correct trials and of error rates, with within-subjects factor TRIAL_TYPE (switch or stay) and between-subjects factor EXPERIMENT (switch or stay experiment), showed that participants responded more slowly (420 vs $290 \mathrm{~ms}$, main effect of TRIAL_TYPE: $F_{(1,19)}=145.894, p<$ $0.001)$ and made more errors $(21.85 \%$ vs $1.40 \%$, main effect of TRIAL_TYPE: $\left.F_{(1,19)}=94.614, p<0.001\right)$ in switch compared with stay trials, confirming the effectiveness of the task manipulation. Behavioral effects did not differ between the two experiments, as indicated by the absence of any other significant effects.

In all three control experiments (M1-control, PMd-control, and IPI), these effects were replicated. Participants responded more slowly $\left(\mathrm{M} 1: t_{(5)}=15.790, p=0.002 ; \mathrm{PMd}: t_{(6)}=3.882, p=\right.$ 0.008; IPI: $\left.t_{(5)}=3.733, p=0.014\right)$ and made more errors $\left(\mathrm{M} 1: t_{(5)}\right.$ $=5.716, p<0.001$; PMd: $t_{(6)}=6.252, p=0.001$; IPI: $t_{(5)}=3.191$, $p=0.025)$ on switch compared with stay trials.

\section{Pre-SMA/M1 paired-pulse TMS during switch and stay trials}

The main question addressed in the present study was whether pre-SMA exerts a context-dependent influence on M1. An ANOVA on the MEP data gathered in the switch and stay experiments, with within-subjects factors PULSE (single vs dual) and $\operatorname{SOA}(75,125,175 \mathrm{~ms})$ and between-subjects factor TRIAL_TYPE (switch vs stay, based on the stimulated trials in the switch and stay experiments, respectively), revealed a significant interaction between TRIAL_TYPE, PULSE, and SOA $\left(F_{(2,18)}=3.533, p=\right.$ 0.039 ) (Fig. $3 a)$ and between TRIAL_TYPE and SOA $\left(F_{(2,18)}=\right.$ $4.734, p=0.021$ ). Post hoc tests revealed that for the switch trials, the dual-pulse MEP differed from the single-pulse baseline only at the $125 \mathrm{~ms} \mathrm{SOA}$ (mean \%MEP $=117.82, t_{(10)}=6.373, p<$ 
(a)
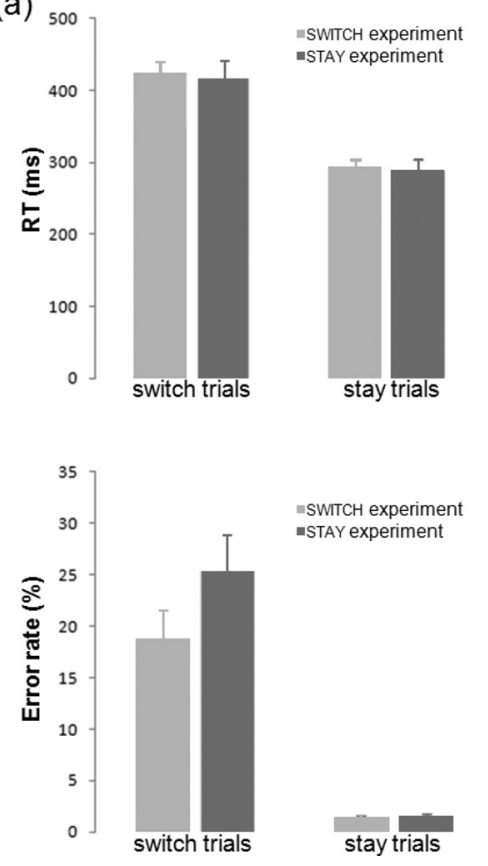

(b)

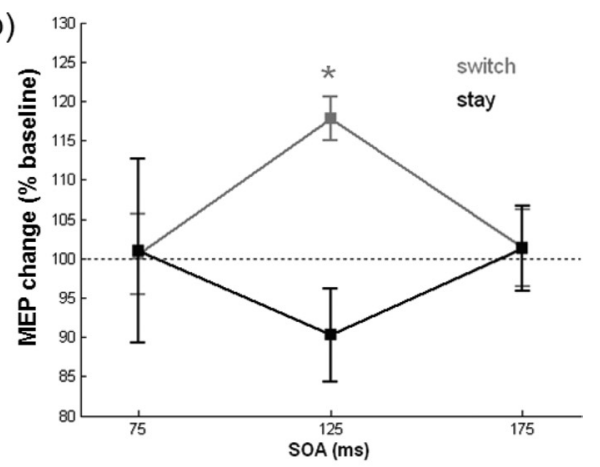

(c)

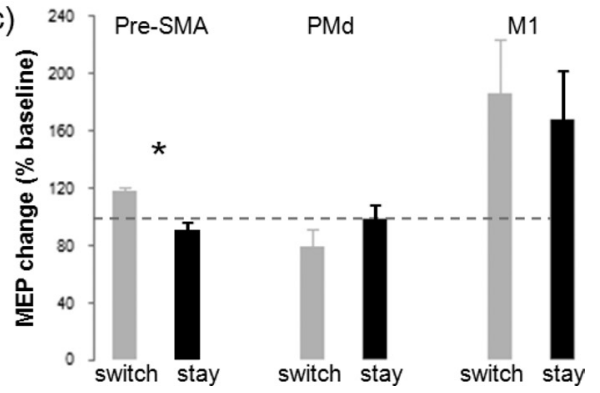

Figure 3. $a$, Behavioral data in the main switch and stay experiments indicate participants reported faster (top) and more accurate (bottom) on switch compared with stay trials. $\boldsymbol{b}$, The effect of pre-SMA conditioning pulses on M1 test pulse-elicited MEP amplitudes was specific to behavioral context and SOA. Pre-SMA/M1 functional connectivity significantly increased on switch trials. * indicates significant modulation of MEP amplitudes in dual-pulse compared with respective single-pulse trials. c, Contextspecific facilitations of MEP amplitude at a SOA of $125 \mathrm{~ms}$ were only present when the conditioning coil was place over pre-SMA and were absent when the conditioning coil was over PMd or M1.

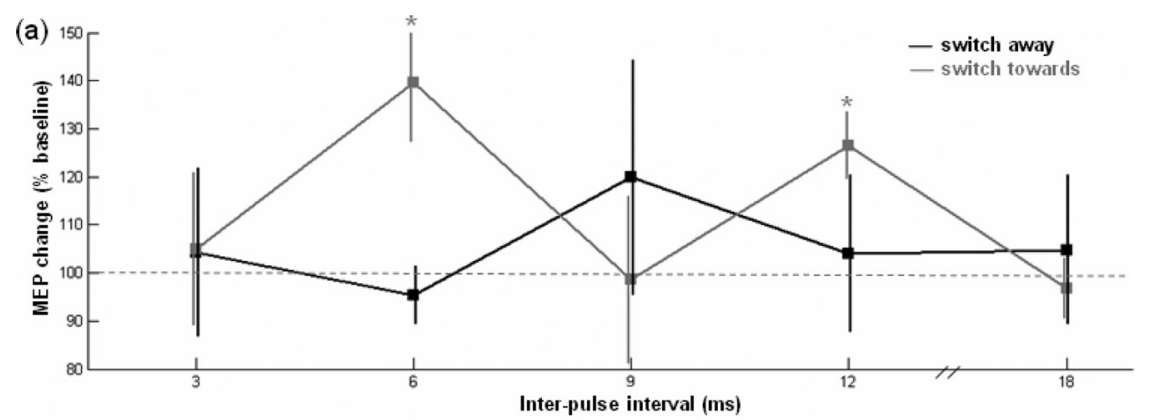

(b)

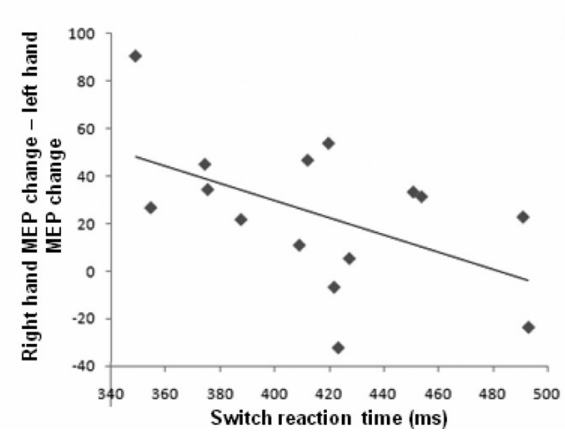

(c)

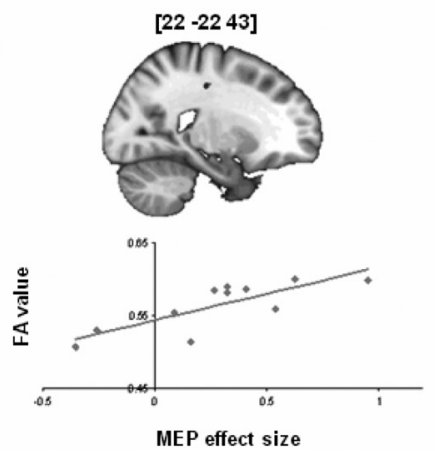

Figure 4. Additional results. $\boldsymbol{a}$, Significant MEP facilitation was seen when switching toward the contralateral hand at 6 and $12 \mathrm{~ms}$ interpulse intervals, but no significant effect was seen when switching to the ipsilateral hand. $\boldsymbol{b}$, A negative correlation was present between the relative facilitation of the contralateral, compared with the ipsilateral, hand and the RT on switch trials.c, Example location of significant correlation between MEP effect size and white matter intensity and scatter plot of individual data within this cluster.

0.001). Furthermore, within the switch experiment, MEP changes at SOA $125 \mathrm{~ms}$ were significantly greater than MEP changes at SOA $75 \mathrm{~ms}\left(t_{(10)}=3.361, p=0.007\right)$ and SOA $175 \mathrm{~ms}$ $\left(t_{(10)}=2.591, p=0.027\right)$. None of these effects reached signifi- cance in the stay trials. It might be argued that switches after a larger number of stay trials were anticipated by the participants. However, the modulatory effect of preSMA TMS on M1 was still present even on such "late" switch trials (supplemental Material IV, available at www.jneurosci. org as supplemental material).

\section{Anatomical specificity}

In the M1-control experiment, the influence of the conditioning pulse over $\mathrm{M} 1$ led to an increase in MEP amplitude $(\% \mathrm{MEP}=176 \%$, main effect of PULSE $\left(F_{(1,5)}=7.731, p=0.039\right)$. However, this influence was no longer context specific; there was no main effect of TRIAL_TYPE $\left(F_{(1,5)}=1.336, p=0.300\right)$ and, crucially, no interaction between TRIAL_TYPE and $\operatorname{PULSE}\left(F_{(1,5)}=0.317, p=0.598\right)$. In the PMd-control experiment, there were no significant effects (main effect of PULSE: $F_{(1,6)}=1.611, p=0.251$; main effect of TRIAL_TYPE: $F_{(1,6)}=2.041, p=0.203$; TRIAL_TYPE $\times$ PULSE: $F_{(1,6)}=4.033$, $p=0.091)$. Although the TRIAL_TYPE $\times$ PULSE interaction approached significance, the effect is in the opposite direction of the effect of pre-SMA, with PMd influence during switch trials trending toward an inhibitory effect on M1. Thus, the facilitatory effect on M1 activity on switch trials is mediated via a pre-SMA/M1 pathway and does not reflect processes internal to M1 or spreading of activation to PMd (Fig. 3c).

To further establish the anatomical specificity of the present effects, we correlated the MEP changes in the switch experiment with individual differences in the white matter integrity of anatomical tracts (Fig. $4 c$; supplemental Material II, available at www.jneurosci.org as supplemental material). This analysis suggested that the effects were mediated solely by pathways underlying premotor and primary motor areas.

\section{Selectivity of pre-SMA/M1 interactions}

We further investigated the specificity of the pre-SMA influence on M1 on switch trials by analyzing data from the switch experiment for trials in which participants switched toward and ultimately selected the hand contralateral to the stimulated M1 (here, right hand), and trials in which participants had prepared, but ultimately inhibited, the hand contralateral to the stimulated M1 and finished the trial by switching to the hand ipsilateral to the stimulated M1. Although there was unambiguous evidence that pre-SMA facilitated the M1 corresponding to the unprepared hand to which subjects were about to switch, evidence for a significant influence of pre-SMA on the M1 corresponding to the 
preprepared hand that was not to respond was less clear-cut (supplemental Material III, available at www.jneurosci.org as supplemental material). The absence of any inhibitory influence on such trials may be considered surprising. A final experiment was run to test whether this effect might be present at other IPIs. This experiment (Fig. 4a) replicated the significant facilitation of MEP size at SOA 125 using a $6 \mathrm{~ms}$ IPI when switching toward the stimulated hand compared with baseline $\left(t_{(5)}=3.193, p=\right.$ $0.024)$. There was no significant modulation, including no inhibitory modulation, of MEPs when switching away from the stimulated hand at any IPI (all $p>0.45$ ). There was also a significant facilitation when switching toward the stimulated hand at a $12 \mathrm{~ms}$ IPI $\left(t_{(5)}=3.726, p=0.014\right)$.

Additionally, there was a significant difference in MEP facilitation in the IPI experiment when switching toward the stimulated compared with the nonstimulated hand at a $6 \mathrm{~ms}$ IPI $\left(t_{(5)}=2.932, p=0.033\right)$. The presence of a hand-selective facilitation effect here but not in the switch experiment might be explained by individual differences in RTs. If the pre-SMA facilitates the correct response, as suggested by the hand difference in the IPI experiment, then the difference between influences on the motor areas might be greater later in the response selection process. Hence, we assessed whether, in the combined data from the switch and IPI experiments, there was evidence that shorter switch RTs were associated with a greater relative facilitation of the contralateral, compared with the ipsilateral hand. After rejection of two outliers using Grubb's test, we indeed found such a negative correlation $(\rho=-0.510$, $p=0.026)$ (Fig. 4b).

\section{Discussion}

Our results show that pre-SMA influences corticospinal excitability at a short latency of $6 \mathrm{~ms}$. The effect is temporally specific to a period $125 \mathrm{~ms}$ after movement instruction, anatomically specific to pre-SMA, and occurs only during action reprogramming.

Previous studies have used paired-pulse TMS to demonstrate increased functional connectivity between premotor and motor regions during the initiation of a sensorimotor transformation (Koch et al., 2006; O'Shea et al., 2007). The current study is the first to employ this technique to study action selection under conditions of response conflict, specifically in the case of the inhibition of a prepared response and the selection of an alternative, a process we refer to as action reprogramming. Action reprogramming recruits a number of neural structures in addition to those involved in normal action selection (Ullsperger and Von Cramon, 2001; Mars et al., 2007), including pre-SMA. Pre-SMA modulation of M1 did not reflect the initiation of a sensorimotor transformation, which was similar on both stay and switch trials; instead, the effects reported reflect a change in expectation, built up over the course of several trials, about which of two sensorimotor transformations should be enacted. The fact that earlier paired-pulse TMS studies (Koch et al., 2006; O'Shea et al., 2007) focused on the initial action selection, whereas the current study focuses on the more complex process of reprogramming an action, might explain why the timing of the effects in the current study were at a later SOA, $125 \mathrm{~ms}$, than in the earlier studies. Furthermore, at the critical SOA the latency (IPI) of influence of pre-SMA over M1 was shorter ( $6 \mathrm{~ms}$ ) than the latency studied in previous PMd/M1 experiments ( $8 \mathrm{~ms}$ ). Our IPI control experiment showed no effects at the latency normally used in PMd/M1 experiments.
The pattern of influence of pre-SMA on M1 reflected whether the stimulated M1 needed to be inhibited or facilitated. Pre-SMA facilitated M1 activity associated with the correct, but unprepared, action. The M1 associated with the incorrect, but prepared, action was not, however, inhibited at any pre-SMA/M1 stimulation latency. It was relatively less facilitated by pre-SMA stimulation than the correct hand $\mathrm{M} 1$, and this relative difference was most prominent in the fastest switchers. This may indicate that pre-SMA normally brings about action reprogramming through a complex combination of facilitatory and inhibitory influences that are not mimicked by the artificial activation patterns induced by TMS, or that pre-SMA is concerned with a higher level of control.

That pre-SMA/M1 effects are reliably seen at latencies as short as $6 \mathrm{~ms}$ in both switch and IPI experiments is important, because it constrains hypotheses about the routes by which pre-SMA might influence M1. Although a basal ganglia/subthalamic route might mediate important aspects of action inhibition (Isoda and Hikosaka, 2008), even the most direct path through this route is associated with neuronal latencies in excess of the $6 \mathrm{~ms}$ preSMA/M1 latency (Nambu et al., 2000). Similar considerations also rule out that a pathway via lateral prefrontal cortex mediated these short-latency effects, even if that pathway is important for regulating longer term changes in cognitive control (Kerns et al., 2004). These observations are consistent with the fact that correlations between white-matter integrity and changes in preSMA/M1 functional connectivity were restricted to dorsomedial frontal cortex.

Previous work has suggested that corticospinal excitability is modulated by recent trial history (Bestmann et al., 2008) and it might be argued that differential switch expectancies, based on the number of preceding stay trials, are what actually influence pre-SMA/M1 functional connectivity. Although the present study was not designed to test such a hypothesis, a preliminary investigation showed that switch-related pre-SMA/M1 effects were apparent even when the switches were very predictable, because many stay trials had elapsed.

In conclusion, the pre-SMA influences M1 at a short latency of $6 \mathrm{~ms}$ during action reprogramming, but not during simple action selection. The effect is specific to the cognitive context, anatomically specific to pre-SMA, and temporally specific, being strongest $125 \mathrm{~ms}$ after the onset of a cue, indicating the need for action reprogramming.

\section{References}

Bestmann S, Harrison LM, Blankenburg F, Mars RB, Haggard P, Friston KJ, Rothwell JC (2008) Influence of contextual uncertainty and surprise on human corticospinal excitability during preparation for action. Curr Biol 18:775-780.

Civardi C, Cantello R, Asselman P, Rothwell JC (2001) Transcranial magnetic stimulation can be used to test connections to primary motor areas from frontal and medial cortex in humans. Neuroimage 14:1444-1453.

Isoda M, Hikosaka O (2007) Switching from automatic to controlled action by monkey medial frontal cortex. Nat Neurosci 10:240-248.

Isoda M, Hikosaka O (2008) Role for subthalamic nucleus neurons in switching from automatic to controlled eye movements. J Neurosci 28:7209-7218.

Kerns JG, Cohen JD, MacDonald AW 3rd, Cho RY, Stenger VA, Carter CS (2004) Anterior cingulate conflict monitoring and adjustments in control. Science 303:1023-1026.

Koch G, Franca M, Del Olmo MF, Cheeran B, Milton R, Alvarez Sauco M, Rothwell JC (2006) Time course of functional connectivity between dorsal premotor and contralateral motor cortex during movement selection. J Neurosci 26:7452-7459. 
Mars RB, Piekema C, Coles MG, Hulstijn W, Toni I (2007) On the programming and reprogramming of actions. Cereb Cortex 17:2972-2979.

Nachev P, Wydell H, O’Neill K, Husain M, Kennard C (2007) The role of the pre-supplementary motor area in the control of action. Neuroimage 36:T155-T163.

Nambu A, Tokuno H, Hamada I, Kita H, Imanishi M, Akazawa T, Ikeuchi Y, Hasegawa N (2000) Excitatory cortical inputs to pallidal neurons via the subthalamic nucleus in the monkey. J Neurophysiol 84:289-300.

O'Shea J, Sebastian C, Boorman ED, Johansen-Berg H, Rushworth MFS (2007) Functional specificity of human premotor-motor interactions during action selection. Eur J Neurosci 26:2085-2095.

Picard N, Strick PL (1996) Motor areas of the medial wall: A review of their location and functional activation. Cereb Cortex 6:342-353.
Rushworth MF, Hadland KA, Paus T, Sipila PK (2002) Role of the human medial frontal cortex in task switching: A combined fMRI and TMS study. J Neurophysiol 87:2577-2592.

Shima K, Mushiake H, Saito N, Tanji J (1996) Role for cells in the presupplementary motor area in updating motor plans. Proc Natl Acad Sci U S A 93:8694-8698.

Taylor PC, Nobre AC, Rushworth MF (2007) Subsecond changes in topdown control exerted by human medial frontal cortex during conflict and action selection: a combined transcranial magnetic stimulationelectroencephalography study. J Neurosci 27:11343-11353.

Ullsperger M, von Cramon DY (2001) Subprocesses of performance monitoring: A dissociation of error processing and response competition revealed by event-related fMRI and ERPs. Neuroimage 14:1387-1401. 